\title{
A list of data for the broadening of metallic lines by neutral hydrogen collisions ${ }^{\star}$
}

\author{
P.S. Barklem ${ }^{1}$, N. Piskunov ${ }^{1}$, and B.J. O'Mara ${ }^{2}$ \\ 1 Uppsala Astronomical Observatory, Box 515, S 751-20 Uppsala, Sweden \\ 2 Department of Physics, The University of Queensland, St Lucia 4072, Australia
}

Received July 20, 1999; accepted January 3, 2000

\begin{abstract}
A list of data for the broadening by neutral hydrogen collisions of many astrophysically important spectral lines, which has been incorporated into the Vienna Atomic Line Database (VALD), is presented. Data for lines of neutral atoms are interpolated from the tabulated data of Anstee \& O'Mara (1995), Barklem \& O'Mara (1997), and Barklem et al. (1998). Data for lines of singly ionised atoms are compiled from the calculations by Barklem \& O'Mara (1998, 2000). The list at present contains data for 4891 lines between 2300 and $13000 \AA$ of elements from $\mathrm{Li}$ to $\mathrm{Ni}$. We examine the statistical impact of the new theory by comparison with the previously available data. We also demonstrate the direct effect on spectral synthesis calculations.
\end{abstract}

Key words: atomic data - line: profiles — astronomical data bases: miscellaneous

\section{Introduction}

Over the past several years, the line broadening theory developed by Anstee \& O'Mara (1991) has been used to compute line broadening cross-sections due to hydrogen collisions for a range of transitions between general states with given effective principal quantum numbers using Coulomb wavefunctions. For $s-p$ and $\mathrm{p}-\mathrm{s}$ transitions these calculations were presented by Anstee \& O'Mara (1995), for $\mathrm{p}-\mathrm{d}$ and $\mathrm{d}-\mathrm{p}$ by Barklem \& O'Mara (1997), and for $\mathrm{d}-\mathrm{f}$ and $\mathrm{f}-\mathrm{d}$ transitions by Barklem et al. (1998).

More recently, the theory has been developed further to allow the calculation of broadening data for specific

Send offprint requests to: P.S. Barklem

* Table 1 is only available in electronic form at the CDS via anonymous ftp to cdsarc.u-strasbg.fr (130.79.128.5) or via anonymous ftp to ftp.astro.uu.se/pub/Spectra/barklem/hlist/ lines of singly ionised elements (Barklem \& O’Mara 1998). It was found that the interatomic interactions could not be considered in the same general way as for neutrals, and hence each line needs to be considered individually. This has been done for astrophysically important lines of the alkaline earth elements, and results have been presented by Barklem \& O'Mara (1998, 2000).

The goal of the work presented in this paper was to produce a list of data based on these calculations for a large number of spectral lines. We aimed in particular to include the majority of strong lines in cool stars, where this broadening mechanism is most important. We have also incorporated this data into the Vienna Atomic Line Database (Kupka et al. 1999) and examined statistically the impact of the new list.

\section{Method}

In order to find the line broadening cross-section for a given line from the tabulated theoretical data, one must correctly determine the effective principal quantum numbers $n^{*}$ and orbital angular momentum quantum numbers $l$ for each state of the transition. To determine $n^{*}$ one must know the binding energy of the optical electron. Often this is just the difference between the ionisation energy for the species (which is defined as that energy required to ionise the ground state) and the energy of the state considered. However if the atom has two excited electrons then this series limit is no longer appropriate, and one must account for the excited parent configuration.

These types of states are common in iron group elements, and a particular example was explained in detail by Barklem et al. (1998). Failure to account for this can lead to quite substantial overestimates of the broadening. An extreme example is the $6439 \AA$ line of neutral calcium, where if one does not account for the excited parent configuration one finds a cross-section of 920 atomic units, while if one accounts for the parent configuration correctly one 


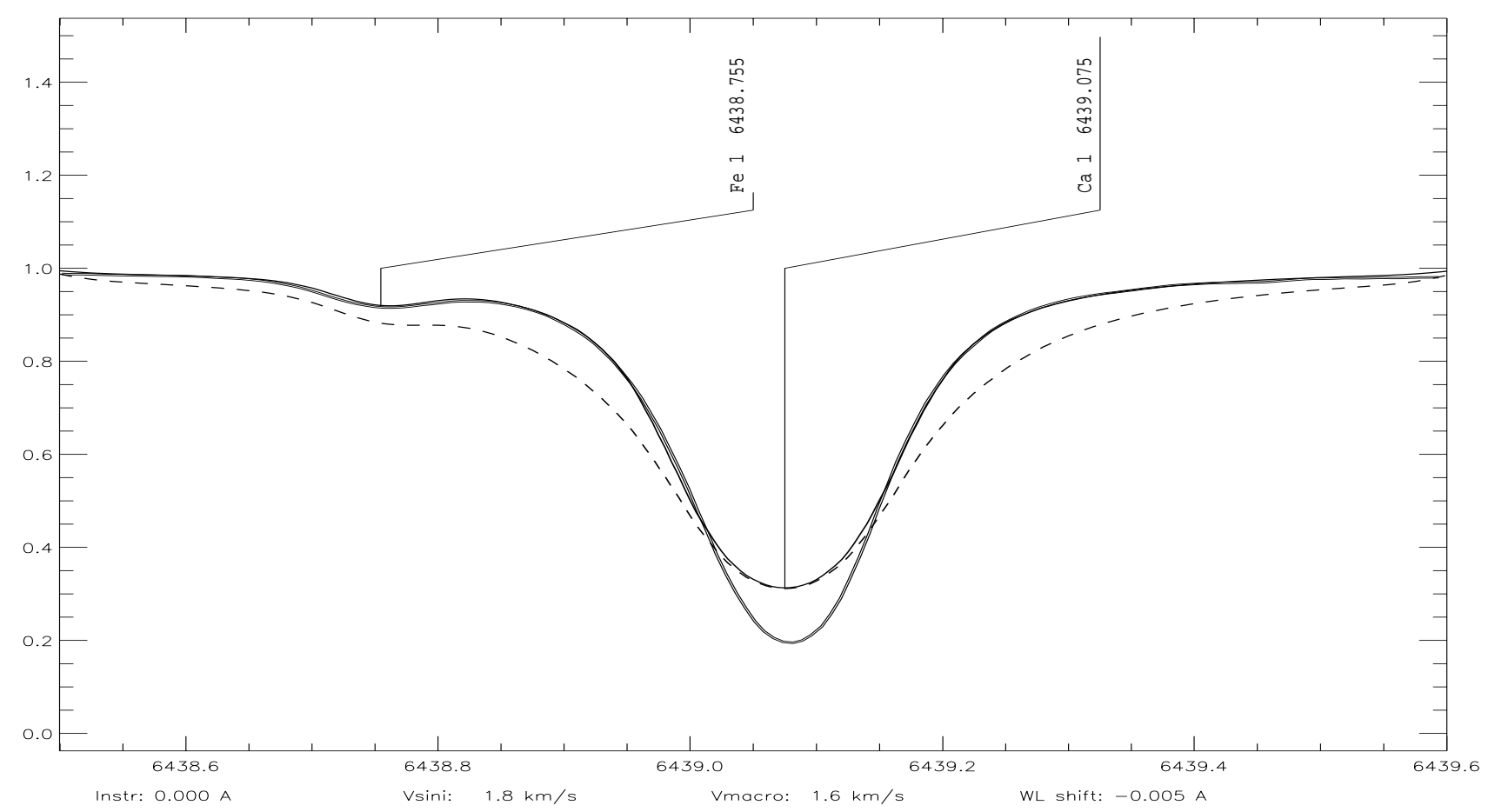

Fig. 1. Comparison of synthetic flux spectra with solar observations (double line - NSO/Kitt Peak data) for the Ca $6439 \AA$ line. Synthetic spectra are shown for cross-sections of 366 (full) and 920 (dashed) atomic units using LTE and the Holweger \& Müller (1974) model atmosphere, meteoritic abundances (Grevesse et al. 1996) and other atomic data from VALD

obtains 366 atomic units. This latter value is completely consistent with the observed broadening in the solar spectrum as shown by Fig. 1 .

Hence to correctly compute $n^{*}$ requires that one knows both the energy of the level and the correct series limit for the parent configuration of the state. If the series limit is not available, the next best thing to have then is detailed information about the electron configuration of the state from which one could determine the parent configuration and series limit.

The Kurucz (1993) CDROM lists of spectral line data, which appear to be the most comprehensive currently available, provide the energy levels but unfortunately do not provide the detail in the electron configuration required. It does provide the term designations which can be converted to electron configurations but requires significant extra work which we may consider doing in the future.

However, the NIST Atomic Spectra Database (http://physics.nist.gov/cgi-bin/AtData/main_asd) does provide complete electron configuration information, and although the database is not as comprehensive as the Kurucz lists it still covers a large number of spectral lines. Most importantly for our purposes, the list covers a large fraction of the lines which attain substantial strength in cool stars where this broadening mechanism will be important. For the purpose of identification and integrating the data into VALD, it is also necessary to have species, wavelengths and $J$ quantum numbers. All of this is also provided by the NIST Atomic Spectra Database.

Data were extracted from the NIST database for lines of all neutral species in the wavelength region between 2300 and $13000 \AA$, where all of the required information was available. This included all elements with atomic number up to and including $28(\mathrm{Ni})$. Data for hydrogen lines have not been included as the broadening is very complex and is currently under investigation. Lines of noble gases have not been included as the model of the interaction is inappropriate. Lines of noble gases are never strong in cool stars in any case. Lines with $\log g f<-5$ were also excluded, as such lines will always be weak in stellar spectra and hence their equivalent widths will be unaffected by this broadening mechanism.

In a previous paper (Barklem et al. 1998) a fortran program was announced which was able to interpolate data from the three sets of tables for lines of neutral atoms. The code was placed on the World-Wide-Web. This code formed the core of the program used to compute the line broadening data in this list. Line data were then computed for all lines from the NIST list where the transition type and the $n^{*}$ values fell on the available tables, which was certainly the majority of the lines. The most difficult part of the processing was the determination of the correct parent configuration. 
A list of approximately 300 lines, which had been previously compiled manually, was merged with the list computed from the NIST data. The manually compiled list contained the strongest and most important lines of neutral species in the solar spectrum, and also included the data for lines of ionised atoms that has been computed to date. This served to fill any important gaps in the NIST line list.

\section{The line list}

The list is given as Table 1 available in electronic form via anonymous ftp at the CDS and UAO ftp servers (see earlier footnote). It contains data for 4891 spectral lines. The data columns in the table are:

1. Packed parameter of form $Z+0.01(Z-N)$, where $Z$ is the atomic number and $N$ is the number of electrons.

2. Air Wavelength $(\AA)$.

3. Lower level $J$ quantum number.

4. Upper level $J$ quantum number.

5. Lower level energy (eV).

6. Upper level energy $(\mathrm{eV})$.

7. Lower level energy $\left(\mathrm{cm}^{-1}\right)$.

8. Lower level series limit $\left(\mathrm{cm}^{-1}\right)$.

9. Upper level energy $\left(\mathrm{cm}^{-1}\right)$.

10. Upper level series limit $\left(\mathrm{cm}^{-1}\right)$.

11. Lower level $l$ quantum number.

12. Upper level $l$ quantum number.

13. Cross-section $\sigma$ for $v=10000 \mathrm{~m} \mathrm{~s}^{-1}$ (a.u.).

14. Velocity parameter $\alpha$.

15. Log of line width (FWHM) per perturber at $T=$ $10000 \mathrm{~K}\left(\mathrm{rad} \mathrm{s}^{-1} \mathrm{~cm}^{3}\right)$.

16. Temperature dependence exponent $(1-\alpha) / 2$.

A short sample of the data between 5327.0 and $5329.5 \AA$ is presented in Table 2. We show later the effects on synthesis of this region.

The broadening data from the list is incorporated into VALD which provides various tools for intelligent retrieval of atomic data, which can be accessed at http://www.astro.univie.ac.at/ vald. The list is currently known as "VALD 2: VanderWaals" under the VALD-2 referencing system, and under the current default configuration for VALD is always used when available. Under VALD-2, for reasons of continuity, the value returned is the logarithm of the full line width at $10000 \mathrm{~K}$, and there is no indication of the temperature dependence. Many spectrum synthesis programs use this value assuming the line width is proportional to $T^{0.3}$ which is the result from the Unsöld theory (1955) using the van der Waals interaction. This would correspond to a velocity parameter of $\alpha=0.4$ in the Anstee \& O'Mara theory as the cross-section is related to temperature by

$\sigma \propto T^{\frac{1-\alpha}{2}}$.

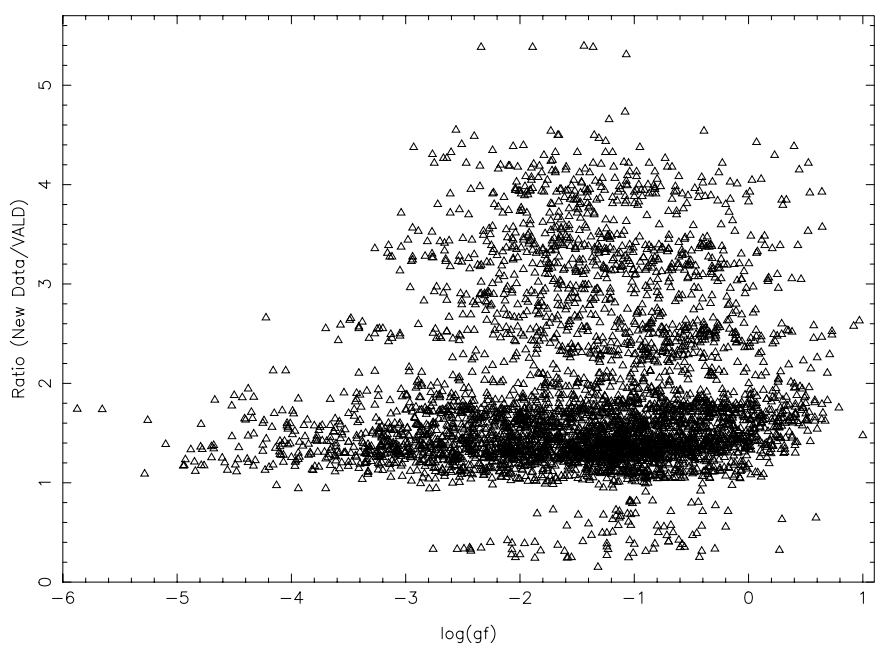

Fig. 2. Comparison of the new data with that otherwise available in VALD. The ratio of the two line widths at $10000 \mathrm{~K}$ is plotted against the $\log (g f)$ value from VALD

Calculations have usually found $\alpha$ to be somewhat lower (between 0.2 and 0.4 ), implying a slightly stronger dependence on temperature, typically of the form $T^{0.38}$. However approximation to $T^{0.3}$ usually has only a small effect on the line shape.

In a future version of VALD, users will be able to choose either the current format or to obtain both the line broadening cross-section and the velocity parameter.

\subsection{List analysis}

We now briefly present some analysis designed to demonstrate the overall impact of the new data.

Data were extracted from VALD using the default configuration but not including this list. That is, the best data otherwise available in VALD. These values otherwise in VALD are those from the Kurucz CDROM line lists, which were mostly computed from the Unsöld theory (some have been corrected with better calculations such as the $\mathrm{Mg} b$ lines discussed later). These values were then compared with the values provided by our new list.

Some 583 lines did not previously have data for broadening by neutral hydrogen available in VALD. For those lines where data were previously available we compared the line widths per perturber at $10000 \mathrm{~K}$. Figures 2 and 3 show the ratio of the new width to the previous VALD line widths, plotted against $\log (g f)$ and excitation respectively. The data show a general increase in the value for the broadening. On average, the line width increases by a factor 1.88 (standard deviation 0.79) when excluding lines with no previous data.

In Fig. 2 the most noticeable feature is the apparent "banding" of the data into three groups. The largest of these groups is the band between the ratios of 1 and 2 . There is then a band at higher ratios and a rather small 
Table 2. Sample of Table 1 for the region 5327.0 to $5329.5 \AA$

\begin{tabular}{|c|c|c|c|c|c|c|c|c|c|c|c|c|}
\hline 26.00 & 5328.038 & 4.0 & 3.0 & 0.914 & 3.239 & 7376.760 & 65572.000 & 26140.178 & 63700.00001 & 239. 0.248 & -7.756 & 0.376 \\
\hline 24.00 & 5328.377 & 4.0 & 5.0 & 2.912 & 5.236 & 23498.840 & 54565.000 & 42261.059 & 54565.00012 & 824. 0.278 & -7.225 & 0.361 \\
\hline 26.00 & 5328.534 & 3.0 & 3.0 & 1.556 & 3.881 & 12560.953 & 65572.000 & 31322.639 & 63700.00001 & 282. 0.252 & -7.685 & 0.374 \\
\hline 22.00 & 5328.728 & 1.0 & 1.0 & 0.812 & 3.137 & 6556.828 & 56045.000 & 25317.813 & 56045.00001 & 305. 0.245 & -7.650 & 0.377 \\
\hline 24.00 & 5329.142 & 4.0 & 4.0 & 2.912 & 5.236 & 23498.840 & 54565.000 & 42258.371 & 54565.00012 & 823. 0.278 & -7.225 & 0.361 \\
\hline
\end{tabular}

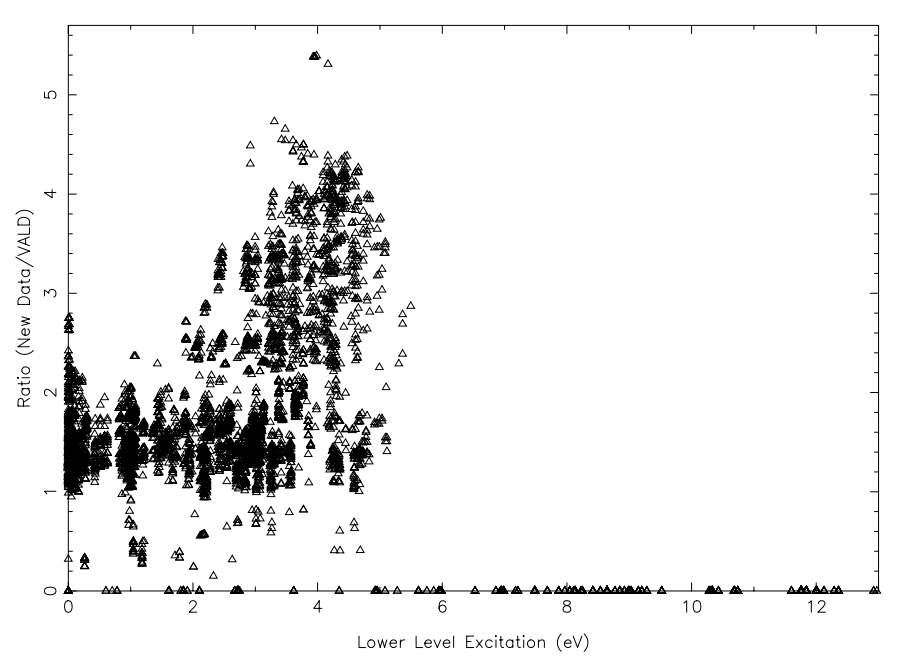

Fig. 3. Comparison of the new data with that otherwise available in VALD. The ratio of the two line widths at $10000 \mathrm{~K}$ is plotted against the lower level excitation. Points on the $x$ axis represent lines for which data were not previously available in VALD

group with ratios less than unity. In Fig. 3, we see that the upper band almost completely consists of lines of higher excitation. The most logical explanation for this is that the calculations using the Anstee \& O'Mara theory treat better the states of higher quantum number $l$, which are often more excited, than the Unsöld theory which makes no distinction between angular momentum states. In Fig. 4, we show the ratio plotted against the transition type, and this plot supports this explanation. It shows that the upper band primarily consists of transitions with upper pand d- states while the middle and lower bands are almost exclusively lines with upper s-states. It also supports the effect first found by Carter (1949) for neutral iron lines in the solar spectrum, that for a given binding energy of the optical electron in the upper state of the transition, lines with an upper s-state are broadened more strongly than those with an upper p-state. Although in Fig. 4 there is no line selection on the basis of the binding energy of the optical electron, lines with a transition index of 2 which correspond to transitions with an upper s-state are generally more strongly enhanced than lines with a transition index of 1 which correspond to lines with an upper p-state. In the specific case of iron lines Anstee et al. (1997) have shown that the new line broadening theory accounts quantitatively for the Carter effect whereas conventional van der Waals theory predicts only a very slight effect which does not match the solar spectrum.

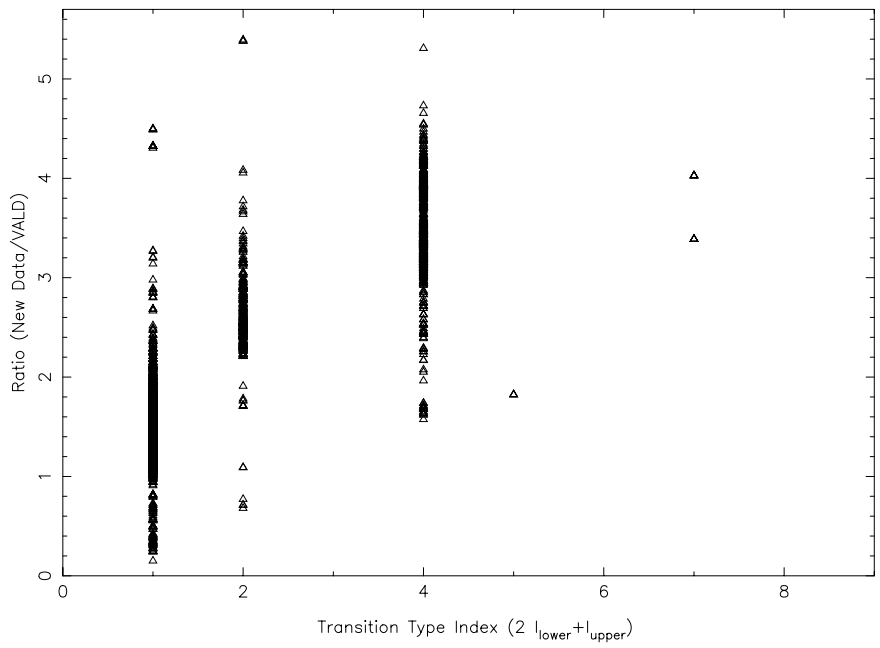

Fig. 4. Comparison of the new data with that otherwise available in VALD. The ratio of the two line widths at $10000 \mathrm{~K}$ is plotted against the transition type indicated by $2 l_{\text {lower }}+l_{\text {upper }}$ where $l_{\text {lower }}$ and $l_{\text {upper }}$ are the lower and upper state $l$ quantum numbers. Hence 1 corresponds to $\mathrm{s}-\mathrm{p}, 2$ to $\mathrm{p}-\mathrm{s}, 4$ to $\mathrm{p}-\mathrm{d}, 5$ to $\mathrm{d}-\mathrm{p}, 7$ to $\mathrm{d}-\mathrm{f}$ and 8 to $\mathrm{f}-\mathrm{d}$. The lack of lines with index 5 and above actually reflects that data were often not previously available for these lines in VALD

The relatively small number of lines making the band with ratios less than unity in Fig. 2 also requires comment. As we see in Fig. 4, this band results almost entirely from $s-p$ transitions. We identified the lines with ratios of less than 0.9 and tried to determine the reason for the result. Notably the $\mathrm{Mg}$ b lines were among these lines. This particular case is explained by the fact that the data in the Kurucz lists for these lines is a corrected value as described by Castelli et al. (1997), not the Unsöld theory result. The remainder of the lines in this band were all lines of V, Cr, Mn, Fe or Co. We checked a number of these lines and found that they are double electron excitations with highly excited parent configurations. We assume that our taking into account these effects is responsible for the differences with the Kurucz calculations.

\subsection{Impact on spectral synthesis}

To further demonstrate the impact of the new data we have selected two spectral regions as examples, and compared synthesis using VALD data in the case where the new list is included with that where the new data is not included. The data have been tested against the solar 


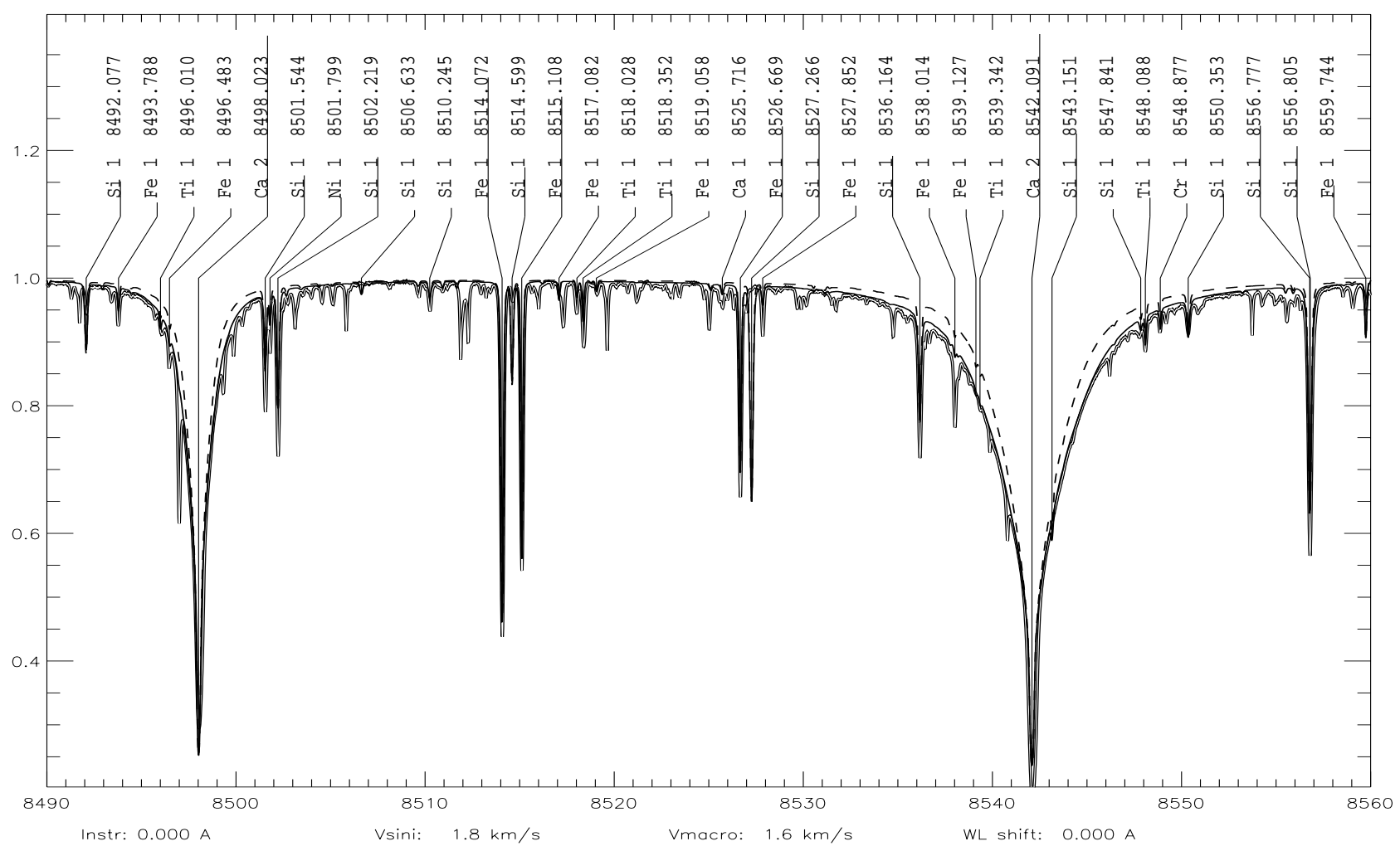

Fig. 5. Plot showing the two bluest lines of the Ca II infrared triplet. Synthetic spectra are shown for VALD with new data (full) and without (dashed) compared to observed (double line) flux spectra (NSO/Kitt Peak Data)

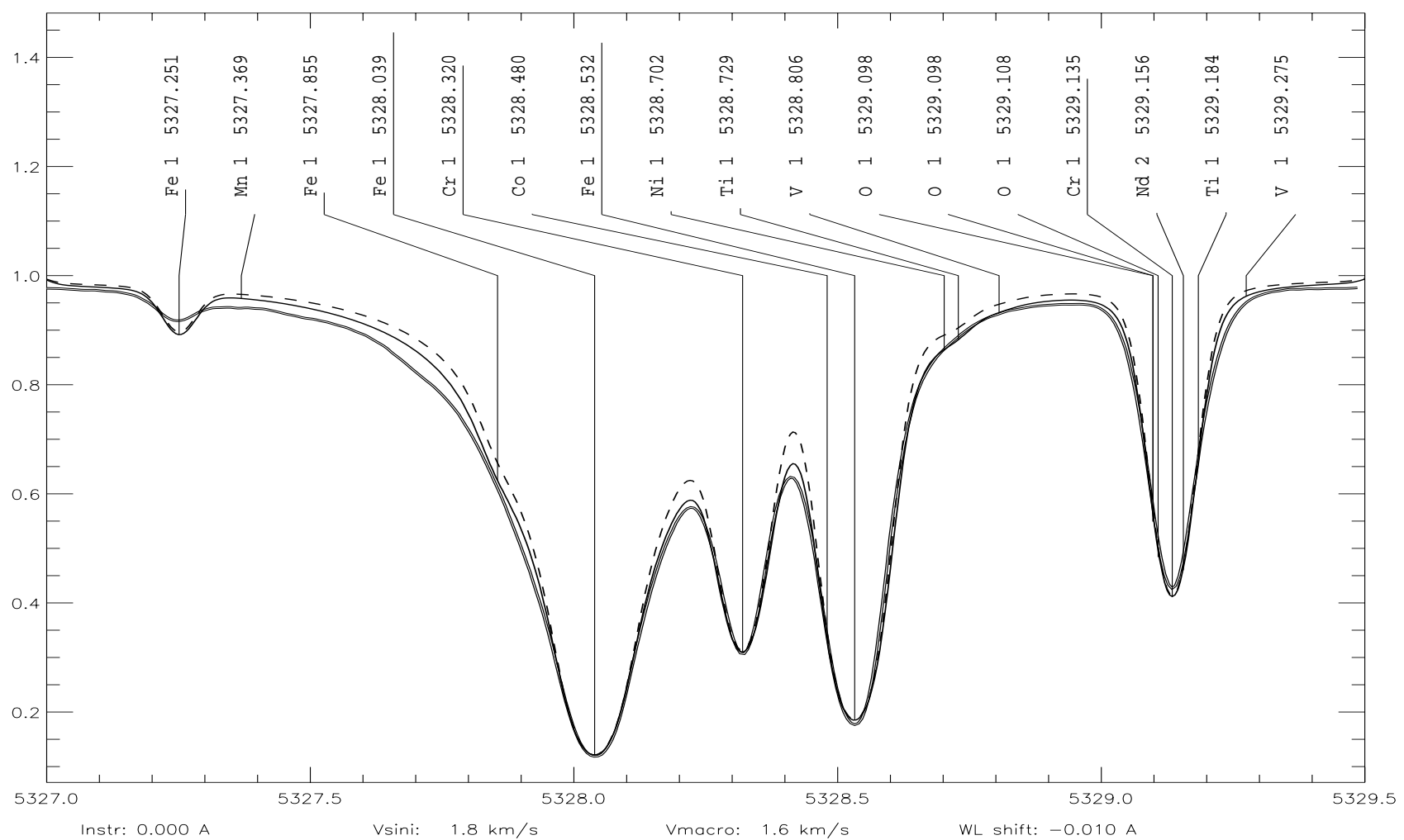

Fig. 6. Plot showing synthetic spectra for the region around the strong line of Fe at $5328 \AA$. Synthetic spectra are shown for VALD with new data (full) and without (dashed) compared to observed (double line) flux spectra (NSO/Kitt Peak Data) 
spectrum in previous papers, hence our aim here is merely to show examples of how the new list effects VALD.

For our synthesis we use the SYNTH code of Piskunov (1992) which assumes LTE. We use the Holweger \& Müller (1974) model atmosphere.

Figures 5 and 6 show two examples of the difference made by inclusion of the new data. In each case we used meteoritic abundances for elements (Grevesse et al. 1996). The Ca II triplet example was chosen as an example where the data makes quite a large positive impact to the VALD data for an important spectral region. The Fe I $5328 \AA$ line region was chosen as example of the impact on a reasonably complex region of the spectrum containing lines of varying strengths. We performed similar comparisons for a number of regions and believe these examples to be representative of the general effect.

In the synthesis of the Fe I $5328 \AA$ region, for the Cr I lines at $5328.3 \AA$ and $5329.1 \AA$ we needed to adjust the wavelengths slightly from those in VALD to match observations. Also, the $\log (g f)$ of the Ti I line at $5328.7 \AA$ needed to be reduced. Both plots show a general improvement using the new data, compared to observation. The fits support the assumption of equality between solar and meteoritic abundances and the new line broadening data within the errors of the analysis.

As part of the transition to VALD-3, we plan to systematically assess the accuracy of spectral lines visible on the Sun by synthesizing the whole spectrum and verifying line identification, accuracy of the central wavelengths, oscillator strengths, broadening parameters and presence of NLTE effects. This project will provide an additional input for the continuation of this work by creating a list of lines that require better quality damping constants.

\section{Comments on accuracy}

\subsection{Physical accuracy}

The papers in which the tables were presented have discussed the accuracy of the data, however some comments are warranted in this context.

While every effort has been made within the context of the model to obtain cross-sections of high computational accuracy, the physical accuracy of the data is difficult to judge. As laboratory data for line broadening by hydrogen are almost non-existent, the only means of testing the data is by application to the solar spectrum.

The most that can be said about the accuracy of the data computed using this method, is that within the accuracy of the $f$-values and synthesis method used, the data (i.e. the tables) have been shown in previous papers to be consistent with the observed broadening in the solar spectrum if one makes the assumption that the solar abundances are equal to the meteoritic ones. We are not aware of any discrepancies. Anstee et al. (1997), in their paper examining the solar Fe abundance using strong lines, did report a discrepancy between the observed broadening and the theoretical broadening for transitions with an upper $\mathrm{e}^{5} \mathrm{D}$ state. However this discrepancy has since been found to be attributable to strong Stark broadening of this energy level. The previous applications of the data to strong lines of a number of elements with well determined $f$-values shows a consistency of derived abundances with meteoritic values that would indicate that the uncertainty may well be as low as $5 \%$.

\subsection{Other error sources}

Perhaps the most likely source of gross error in the list would be mistaken identification of transition types (i.e. angular momentum quantum numbers $l$ ), and incorrect identification of the parent configuration, in the automated processing of the electron configurations. However, comparison with the manually produced list, of which a reasonable fraction of the lines involve double electron excitations, with lines computed automatically revealed no discrepancies.

In any case, all data used in the calculation of the line broadening data, such as angular momentum quantum numbers, energy levels, and series limits, are provided in Table 1 (although this information is not in VALD). Hence, if one suspects an error, the input data can be checked.

\section{Concluding remarks}

Comments on the list, or reports of errors are welcomed, and can be directed to Paul.Barklem@astro.uu.se. We also welcome any suggested additions to the list.

Acknowledgements. Thanks to Friedrich Kupka for his help with integrating this list into VALD, and useful discussions. PB also acknowledges useful discussions with Martin Asplund and Bengt Edvardsson regarding lines of astrophysical importance. PB acknowledges the support of the Swedish Natural Science Research Council. NSO/Kitt Peak FTS data used here were produced by NSF/NOAO.

\section{References}

Anstee S.D., O'Mara B.J., 1991, MNRAS 253, 549

Anstee S.D., O'Mara B.J., 1995, MNRAS 276, 859

Anstee S.D., O'Mara B.J., Ross J.E., 1997, MNRAS 284, 202

Barklem P.S., Anstee S.D., O'Mara B.J., 1998, PASA 15(3), 336

Barklem P.S., O'Mara B.J., 1997, MNRAS 290, 102

Barklem P.S., O'Mara B.J., Ross J.E., 1998, MNRAS 296, 1057

Barklem P.S., O'Mara B.J., 1998, MNRAS 300, 863

Barklem P.S., O'Mara B.J., 2000, MNRAS 311, 535

Carter W.W., 1949, Phys. Rev. 76, 962

Castelli F., Gratton R.G., Kurucz R.L., 1997, A\&A 318, 841 
Grevesse N., Noels A., Sauval A.J., 1996, in Holt S.S., Sonneborn G. (eds.), ASP Conf. Ser. 99, Cosmic Abundances. Astron. Soc. Pac., San Francisco, p. 117

Holweger H., Müller E.A., 1974, Sol. Phys. 39, 19

Kupka F., et al., 1999, A\&AS 138, 119

Kurucz R.L., 1993, CDROM Line Lists
Piskunov N., 1992, Stellar Magnetism - Proceedings of the international meeting on the problem Physics and Evolution of Stars, St. Petersberg, p. 92

Unsöld A., 1955, Physik der Stern Atmosphären, Zweite Auflage. Springer-Verlag 